\title{
Collisionless electrons in a thin high Mach number shock: dependence on angle and $\beta$
}

\author{
M. Gedalin, E. Griv \\ Department of Physics, Ben-Gurion University, Beer-Sheva, 84105 Israel \\ Received: 8 February 1999 / Revised: 12 May 1999 / Accepted: 18 May 1999
}

\begin{abstract}
It is widely believed that electron dynamics in the shock front is essentially collisionless and determined by the quasistationary magnetic and electric fields in the shock. In thick shocks the electron motion is adiabatic: the magnetic moment is conserved throughout the shock and $v_{\perp}^{2} \propto B$. In very thin shocks with large cross-shock potential (the last feature is typical for shocks with strong electron heating), electrons may become demagnetized (the magnetic moment is no longer conserved) and their motion may become nonadiabatic. We consider the case of substantial demagnetization in the shock profile with the small-scale internal structure. The dependence of electron dynamics and downstream distributions on the angle between the shock normal and upstream magnetic field and on the upstream electron temperature is analyzed. We show that demagnetization becomes significantly stronger with the increase of obliquity (decrease of the angle) which is related to the more substantial influence of the inhomogeneous parallel electric field. We also show that the demagnetization is stronger for lower upstream electron temperatures and becomes less noticeable for higher temperatures, in agreement with observations. We also show that demagnetization results, in general, in non-gyrotropic down-stream distributions.
\end{abstract}

Key words. Interplanetary physics (interplanetary shocks; planetary bow shocks)

\section{Introduction}

It is widely believed, (Feldman et al., 1982; Goodrich and Scudder, 1984; Feldman, 1985; Thomsen et al., 1987; Schwartz et al., 1988; Balikhin et al.,1993; Geda-

Correspondence to: M. Gedalin, e-mail: gedalin@bgumail.bgu.ac.il lin et al., 1995b, 1998a; Gedalin and Balikhin, 1998) that electron dynamics in the shock front is determined by the (quasi)static electric and magnetic field in the shock transition layer. In this picture, each electron, while crossing the ramp, acquires the same amount of energy $e \phi^{H T}$ in the de Hoffman-Teller frame (where the upstream plasma velocity is along the upstream magnetic field), $\phi^{H T}$ being the cross-shock potential. The downstream distribution formed as a result of this collisionless dynamics depend on the particular mechanism of the energy redistribution among the perpendicular (with respect to the local magnetic field) and parallel degrees of freedom. If the spatial scale of the magnetic- and electric-field variations inside the ramp is not too small, the electron dynamics is expected to be adiabatic, that is the electron magnetic moment is conserved throughout the shock and $v_{\perp}^{2} / B=$ const (Feldman et al., 1982; Goodrich and Scudder, 1984; Feldman, 1985, Scudder et al., 1986b; Scudder, 1995). In very thin shocks (Newbury and Russell, 1996) the adiabaticity may break down, so that electrons become demagnetized (atleast partially), that is, the magnetic moment is no longer conserved, and a more substantial part of the energy may be transferred into the perpendicular degree of freedom (Balikhin et al., 1993; Balikhin and Gedalin, 1994; Gedalin et al., 1995, 1998a; Ball and Galloway, 1998). In our investigation we use the ratio $v_{\perp}^{2}\left|\mathbf{B}_{0} / v_{\perp, 0}^{2}\right| \mathbf{B} \mid$ along the electron trajectory (if applied) as a quantitative measure of demagnetization. Here $v_{\perp}$ is the perpendicular electron velocity in the point where the total magnetic field has the value $|\mathbf{B}|$, and subscript 0 refers to initial values of these two variables.

While in most of the observed shocks the spatial scales ensure at least approximate conservation of the magnetic moment, there are certainly high Mach number shocks (empirically - with the Mach number roughly $M=V_{u} / v_{A}>3$ ) where adiabaticity is broken because of their narrow front (Newbury and Russell, 1996). For a number of shocks the relation $v_{\perp}^{2} / B=$ const is inconsistent with the width of the downstream distribution 
and $T_{\perp, d} / T_{\perp, u}$ by far exceeds $B_{d} / B_{u}$ (where $u$ and $d$ refer to upstream and downstream, respectively), which is not satisfactorily explained by the adiabatic mechanism (Schwartz et al., 1988). While the adiabatic regime is studied comprehensively (Hull et al., 1998; Gedalin and Griv, 1999) and the dependence on the shock Mach number $M=V_{u} / v_{A}$, angle between the shock normal and upstream magnetic field $\theta$, and upstream electron $\beta_{e}=8 \pi n_{e} T_{e} / B_{u}^{2}$ is determined easily, the corresponding dependencies for the nonadiabatic case are not analyzed so far. Previous studies dealt with the dependence on the cross-shock potential and electron temperature for perpendicular geometry (Balikhin and Gedalin, 1994; Gedalin et al., 1995), local criteria of demagnetization (Balikhin et al., 1998), and mapping of upstream distribution to the downstream distribution (Gedalin and Balikhin, 1998; Gedalin et al., 1998a). However, the dependencies of the demagnetization on $\theta$ and $\beta$ (in the oblique case) have not been analyzed so far. In the present paper we fill this gap considering the electron motion in the thin shock for different shock parameters. The paper is organized as follows. In Sect. 2 we describe the model shock profile used afterwards for numerical analysis of electron trajectories in Sect. 3. We discuss possible implications of the found features of electron behavior for observations in Sect. 4 .

\section{Model shock profile}

Our method is to trace collisionless electron trajectories in a model shock profile in order to establish the connection between the upstream and downstream electron parameters. This task requires to specify magnetic and electric fields in the model of the shock front. In previous analyses (Gedalin et al., 1995a, b, 1998a; Gedalin and Balikhin, 1998) a simple monotonic magnetic field profile was used. Newbury et al. (1998) have shown that small-scale structure in the form of several successive magnetic field jumps is quite typical for the high Mach number shock front. In the present paper we use the following analytical approximation to describe a structured shock profile:

$\frac{B_{z}}{B_{u z}}=\frac{R+1}{2}+\frac{R-1}{2} \tan \mathrm{h}\left[\frac{x}{D}+\left(\frac{x}{D}\right)^{3}+a \sin (2 \pi c x / D)\right]$.

Here the shock normal is along $x$ axis, and the noncoplanarity direction is along $y$ axis, so that the upstream magnetic field in the $x-z$ plain, while the shock front is in the $y-z$ plane. The main $\left(B_{z}\right)$ magnetic field compression $R=B_{d z} / B_{u z}$ is related to the magnetic compression ratio $B_{d} / B_{u}$ as follows: $B_{d} / B_{u}=$ $\sqrt{R^{2} \sin ^{2} \theta+\cos ^{2} \theta}$, where $\theta$ is the angle between the shock normal and upstream magnetic field. In the following numerical analysis $B_{d} / B_{u}=3.5, a=0.6$, and $c=1.3$. The Alfvenic shock Mach number is chosen $M=6$. The obtained profile resembles profiles of some high Mach number shocks (Newbury et al., 1998), and approximately models the magnetic field profile of the shock considered by Gedalin et al. (1998b). Observations (Newbury et al., 1998) show that existence of small-scale (down to $\sim 0.1 \mathrm{c} / \omega_{p i}$ ) large amplitude features is quite typical for high Mach number shocks. Gedalin et al. (1998b) argue that these fine-scale structures smoothes the reflected and gyrating ion distributions and is necessary for maintaining shock stability. So far it is not known what is the mechanism of formation of this small-scale structure nor what determines its scale. It is worth mentioning that from the point of view of electron dynamics single narrow ramp is equivalent to the structured shock front, once the steepest features have the same spatial scale. We choose to study the structured shock front since this profile agrees better with recent observations.

The non-coplanar magnetic field $B_{y}$ is modelled using the following relation (Jones and Ellison, 1987, 1991; Gedalin, 1996; Newbury et al., 1997):

$B_{y}=f \frac{c \cos \theta}{\omega_{p i}} \frac{\mathrm{d} B_{z}}{\mathrm{~d} x}$,

where $f=0.5$ is chosen to retain $B_{y}$ small relative to the main magnetic field component $B_{z}$, even for narrow features, in agreement with observations.

The corresponding de Hoffman-Teller cross-shock electric field is chosen in agreement with Schwartz et al. (1988), Hull et al. (1998), Gedalin et al. (1998b):

$\varphi(x)=\varphi_{0} \frac{|\mathbf{B}|(x)-B_{u}}{B_{d}-B_{u}}$,

where $\varphi_{0}$ is the total cross-shock potential. This relation was originally proposed for adiabatic electrons with the temperature $T \propto|\mathbf{B}|$ (approximately follows from the magnetic moment conservation) and observationally found $n \propto|\mathbf{B}|$ (Scudder et al., 1986a), and later used as a general empirical approximation by Hull et al. (1998). In a self-consistent problem the feedback of electron dynamics on the cross-shock potential should be taken into account. In the present paper, however, we adopt the test particle approach which does not require selfconsistency. Moreover, as we shall see below, the electron distributions inside the shock front, formed due to the electron dynamics in the electric and magnetic fields, have a large gap in the distribution (lack of electrons with $v_{\|}^{2}+v_{\perp}^{2}<2 e \varphi / m_{e}$ ) which has to be filled due to some pre-existing electron population (Feldman et al., 1982; Feldman, 1985), or instabilities resulting in the relaxation and smoothing of the distribution (Veltri et al., 1990, 1992; Veltri and Zimbardo, 1993a, b; Gedalin, 1999), or any other mechanism which is unknown yet (Scudder, 1995; Hull et al., 1998). All this may well affect the relation between the electron distribution and the potential and be responsible for the consistency of the chosen profiles. These questions are beyond the scope of the present paper, where our task is only to study the effects related to the collisionless electron dynamics in the stationary electric and magnetic fields of the narrow shock front.

For the present analysis we chose $e \varphi_{0} /\left(m_{i} V_{u}^{2} / 2\right)=$ 0.15 , which is typical for shocks with strong electron 
heating (Schwartz et al., 1988). The model profiles (total magnetic field, noncoplanar magnetic field, and electric field) for two different $\theta=80^{\circ}$ and $\theta=60^{\circ}$ are shown in Fig. 1, where the coordinate $x$ is measured in the upstream ion convective gyroradii $V_{u} / \Omega_{u}, \Omega_{u}=e B_{u} / m_{i c}$, and the motional electric field $E_{y}=V_{u} B_{u} \sin \theta / c$.

The total ramp width (including the small-scale structure) is $0.4\left(c / \omega_{p i}\right)$, while the scale of the substructure is $\sim 0.1\left(c / \omega_{p i}\right)$, which is in agreement with observations (Newbury et al., 1998). For such narrow small-scale structures the electron motion is expected to be nonadiabatic (Gedalin et al., 1995b, Gedalin and Balikhin, 1998).

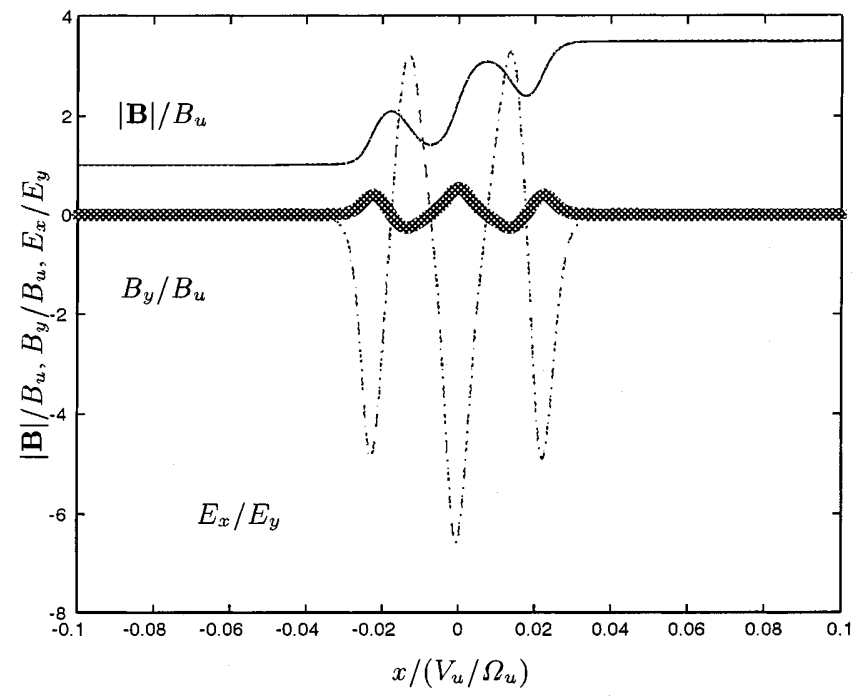

(a)

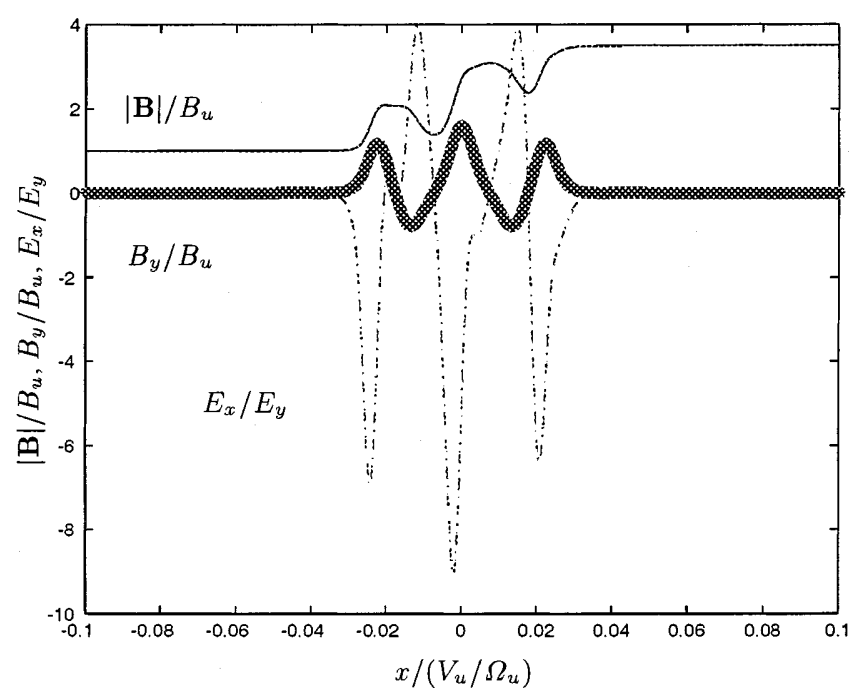

(b)

Fig. 1. Normalized total magnetic field $|\mathbf{B}| / B_{u}$, noncoplanar magnetic field $B_{y} / B_{u}$, and cross-shock electric field $E_{x} / E_{y}$, for (a) $\theta=80^{\circ}$ and (b) $\theta=60^{\circ}$

\section{Electron motion in the model shock profile}

For the analysis of the electron motion in the model shock profile we assume that the upstream electron distribution is Maxwellian. We study two cases of different upstream electron temperatures: $\beta_{e}=0.05$ (stronger demagnetization) and $\beta_{e}=0.25$ (weaker demagnetization), since the effect of demagnetization (breakdown of the relation $v_{\perp}^{2} /|\mathbf{B}|=$ const) should be more pronounced for low $\beta_{e}$ and disappears for high $\beta_{e}$ (Schwartz et al., 1988; Balikhin and Gedalin, 1994; Gedalin et al., 1998a). As previously mentioned we use the ratio $K=v_{\perp, f}^{2}|\mathbf{B}|_{i} / v_{\perp, i}^{2}|\mathbf{B}|_{f}$ as a quantitative measure of demagnetization. Here $i$ and $f$ refer to the beginning and the end of the electron tracing.

We start the study with the motion of a single electron with the initial velocity $\mathbf{v}_{i}=\left(V_{u}, 0, V_{u} \tan \theta\right)$ in the de Hoffman-Teller frame. The upstream velocity of this electron is along the upstream magnetic field, $v_{\perp} \equiv 0$, and in the adiabatic case it remains so throughout the shock. Figure 2 shows the motion of the electron $\left(x, v_{x}\right)$ for different angles between the shock normal and upstream magnetic field, while other shock parameters are retained the same. We cannot use the ratio $K$ in this case, since $v_{\perp, i}=0$. The natural measure of the demagnetization is the final perpendicular velocity of the electron $v_{\perp, f}$. It is clearly seen that the demagnetization is stronger for smaller angles: the down-stream perpendicular velocity is higher for smaller angles and rapidly decreases with the increase of $\theta$. The difference between the cross-shock potential in the normal incidence and de Hoffman-Teller frames $\Delta \varphi=V_{u} \tan \theta \int B_{y} \mathrm{~d} x / c$ is approximately $\propto \sin ^{2} \theta$ for the chosen field profile common for all analyzed cases, which means that the normal incidence frame potential is lower for lower angles. The stronger demagnetization is due to the effects of the larger inhomogeneous parallel electric field $\mathbf{E} \cdot \mathbf{B} /|\mathbf{B}|$ in the more oblique cases, as found by Gedalin et al. (1995b).

The same dependence is seen in Fig. 3 showing trajectories $\left(x, v_{x}\right)$ of 100 electrons in the same shock profiles for the incident Maxwellian with $\beta_{e}=0.05$. The distribution is wider in $v_{x}$ direction for smaller angles. The panels for $\theta=60^{\circ}$ and $\theta=50^{\circ}$ show typical behavior of demagnetized electrons: strong acceleration across the magnetic field (in $x$ direction) in the regions where $-\mathrm{d} E_{x} / \mathrm{dx}$ is sufficiently large, with subsequent gyration (Gedalin et al., 1995b) in the region where adiabaticity is restored. Such demagnetization occurs at all three sub-jumps inside the ramp but its effect is more clearly seen at the first sub-ramp where the electron temperature is still low.

Further information can be obtained from consideration of the collisionless Liouville mapping. We start with the forward mapping where we find the correspondence $\left(v_{\|, u}, v_{\perp, u}\right) \rightarrow\left(v_{\|, d}, v_{\perp, d}\right)$. In this approach the electron distribution is assumed to be gyrotropic, which is definitely correct for the upstream distribution and should be correct for the spatially averaged downstream distribution as well. Figure 4 shows this mapping for 50 

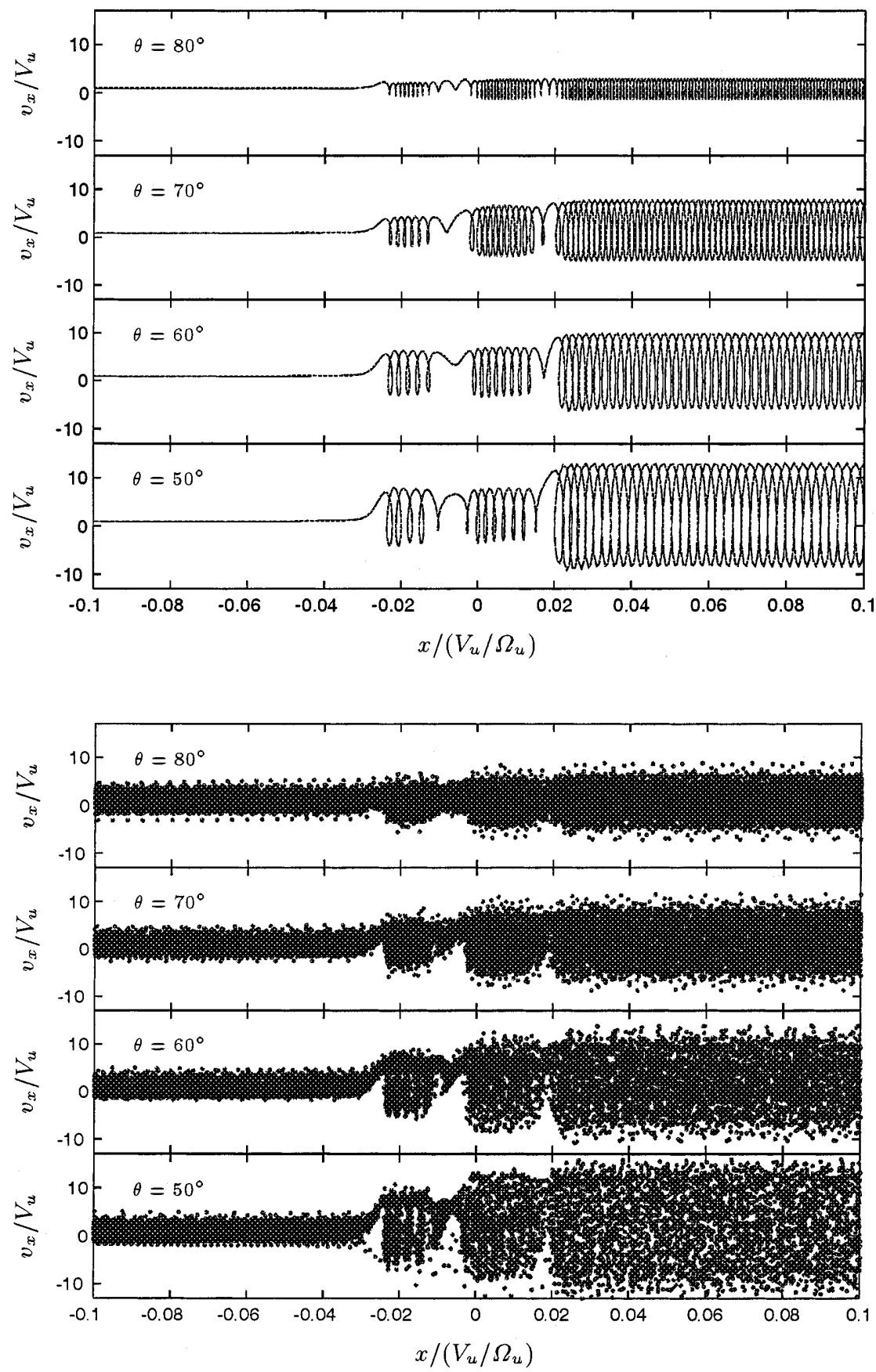

Fig. 2. Trajectory of a single electron with the initial velocity $\mathbf{v}_{i}=\left(V_{u}, 0, V_{u} \tan \theta\right)$ in the de Hoffman-Teller frame for several values of the angle between the shock normal and upstream magnetic field. The Mach number $M=6$, magnetic compression $B_{d} / B_{u}=3.5$, de Hoffman-Teller cross-shock potential $e \varphi_{0}=0.15\left(m_{i} V_{u}^{2} / 2\right)$, and shock width $\approx 0.4\left(c / \omega_{p i}\right)$ are the same for all cases
Fig. 3. Trajectories of 100 electrons for the same shocks as in Fig. 2. The upstream electron distribution is Maxwellian with $\beta_{e}=0.05$ electrons and three different angles between the shock normal and upstream magnetic field: $\theta=80^{\circ}$ (crosses), $\theta=70^{\circ}$ (circles), and $\theta=60^{\circ}$ (stars). In the plot $v_{\|, u}-v_{\|, d}$ the points corresponding to the lower angles are more scattered: while $v_{\|, d}$ varies only slightly for $\theta=80^{\circ}$, the downstream parallel velocities for $\theta=60^{\circ}$ span almost the whole allowed range. Similar behavior is seen in the $v_{\perp, u}-v_{\perp, d}$ plot. For $\theta=80^{\circ}$ there is approximate dependence $v_{\perp, d} / v_{\perp, u} \approx \sqrt{3.5}$, that is, close to the adiabatic regime. In the case $\theta=60^{\circ}$ the downstream perpendicular velocities are substantially scattered over the whole range for the same $v_{\perp, u}$ which corresponds to the loss of the one-to-one mapping $v_{\perp, u} \rightarrow v_{\perp, d}$ for the nonadiabatic regime (Gedalin et al., 1995b). This perpendicular velocity spread is another qualitative measure of demagnetization: larger spread corresponds to stronger deviations from the proportionality $v_{\perp}^{2} \propto|\mathbf{B}|$. The spread of the parallel velocities is closely related to the spread of perpendicular velocities because of the energy conservation: $v_{\perp, d}^{2}+v_{\|, d}^{2}=$ $v_{\perp, u}^{2}+v_{\|, u}^{2}+2 e \varphi_{0} / m_{e}$. Respectively, plot $v_{\|, d}-v_{\perp, d}$ shows that downstream electrons have typically higher perpendicular velocities and lower parallel velocities for smaller angles between the shock normal and upstream magnetic field. The last $v_{\|, u}-v_{\perp, u}$ plot shows that the upstream electron distribution is more subsonic (higher $\left.v_{T e} \cos \theta / V_{u}\right)$ for smaller angles. Here $v_{T e}=\left(T_{e u} / m_{e}\right)^{1 / 2}$ is the upstream electron thermal velocity. 

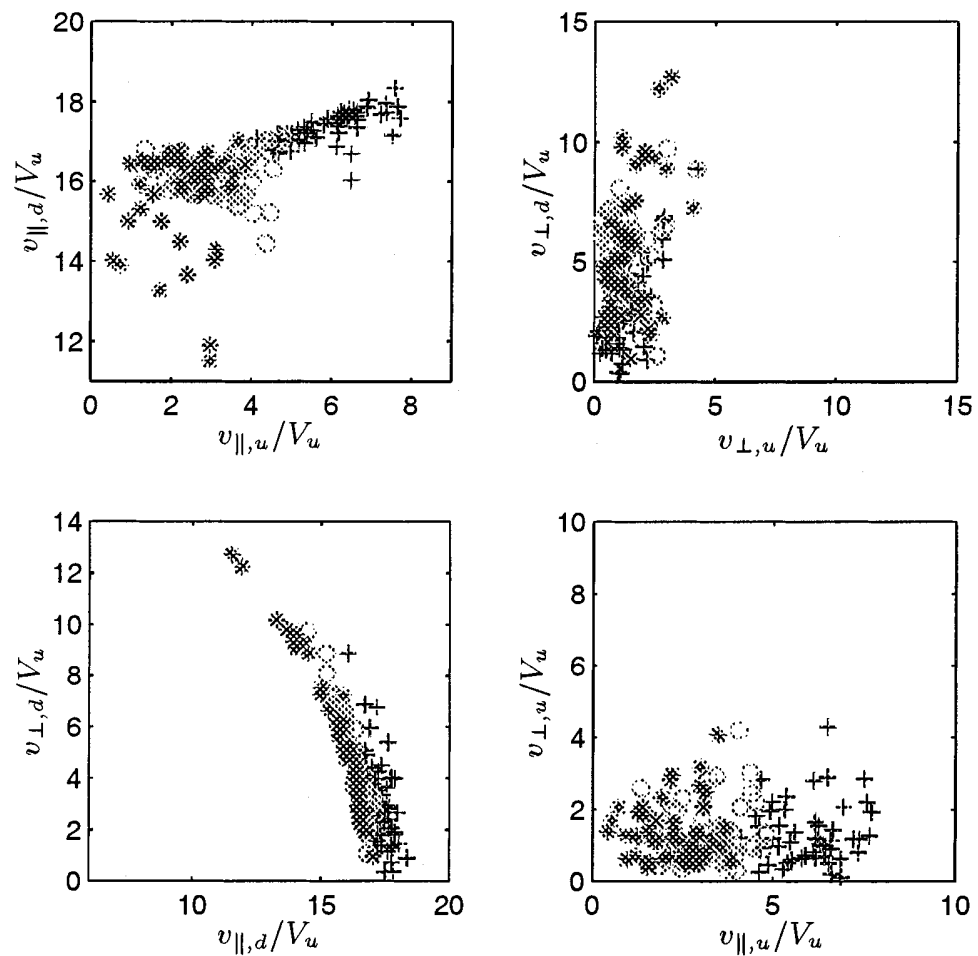

Fig. 4. Liouville mapping for 50 electrons (initially Maxwellian distributed with $\beta_{e}=0.05$ ) for $\theta=80^{\circ}$ (crosses), $\theta=70^{\circ}$ (circles), and $\theta=60^{\circ}$ (stars)

Figure 5 shows the same mapping but for higher upstream electron temperature $\beta_{e}=0.25$. It is clearly seen that the demagnetization is much less pronounced: the scattering of the points in $v_{\|, u}-v_{\|, d}$ and $v_{\perp, u}-v_{\perp, d}$ plots is much weaker. In the $v_{\|, d}-v_{\perp, d}$ plot there is almost no difference between the three cases. In the $v_{\perp, u}-v_{\perp, d}$ plot the points are much more close to the proportionality $v_{\perp, d} / v_{\perp, u} \approx \sqrt{B_{d} / B_{u}}$ than in the corre- sponding plot in Fig. 4. Again the scattering and deviations from this proportionality are more substantial for smaller angles.

Direct quantitative estimate of the degree of demagnetization can be obtained by the comparison of $K=B_{u} v_{\perp, d}^{2} / B_{d} v_{\perp, u}^{2}$ for different angles and $\beta_{e}$, is shown in Fig. 6. Values of $K$ substantially greater than unity correspond to strong demagnetization. Some electrons
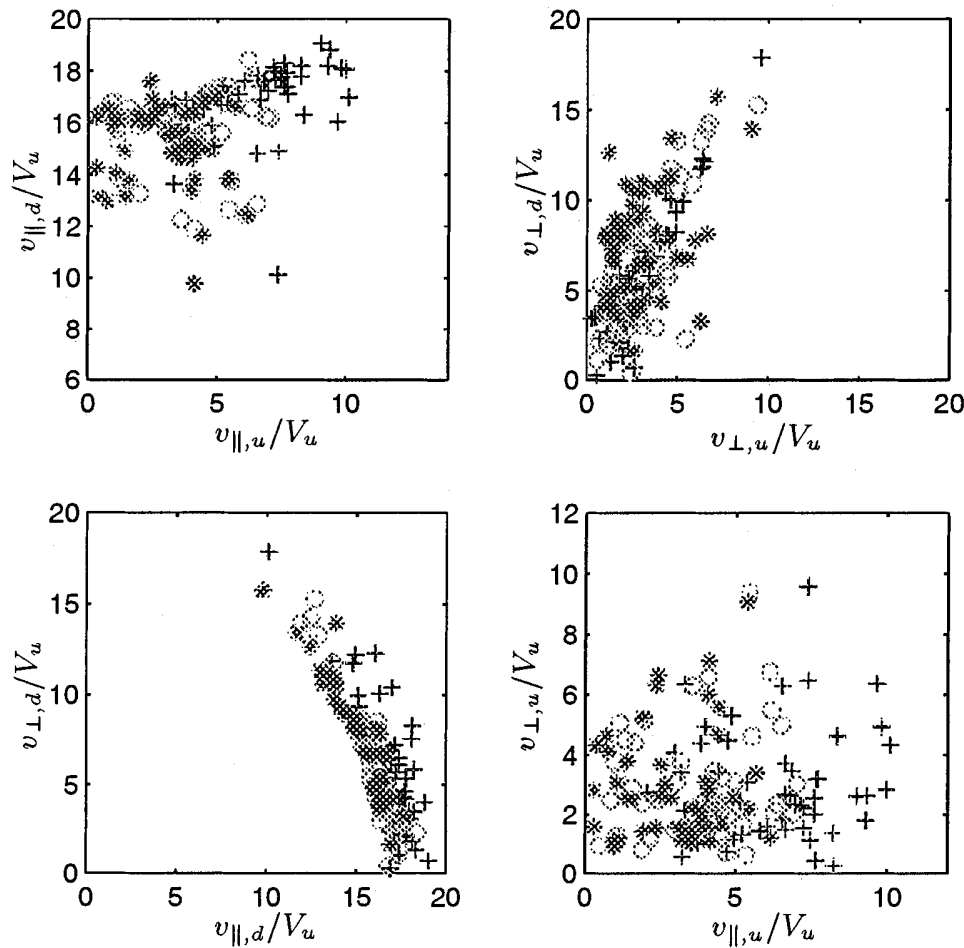

Fig. 5. Liouville mapping for 50 electrons (initially Maxwellian distributed with $\beta_{e}=0.25$ ) for $\theta=80^{\circ}$ (crosses), $\theta=70^{\circ}$ (circles), and $\theta=50^{\circ}$ (stars) 


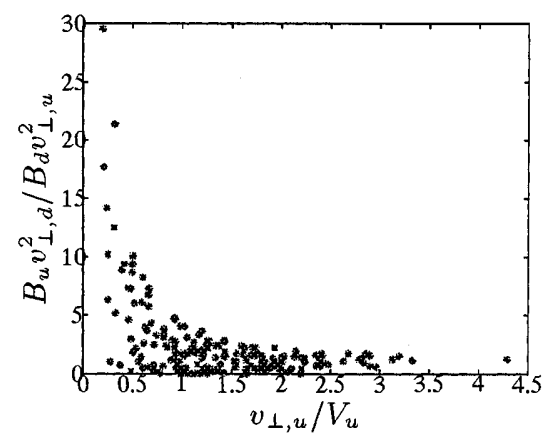

(a)

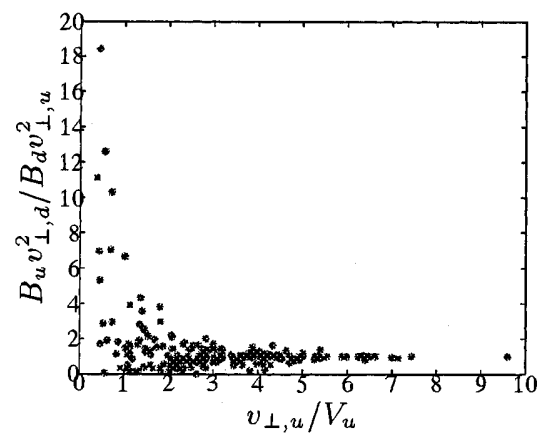

(c)

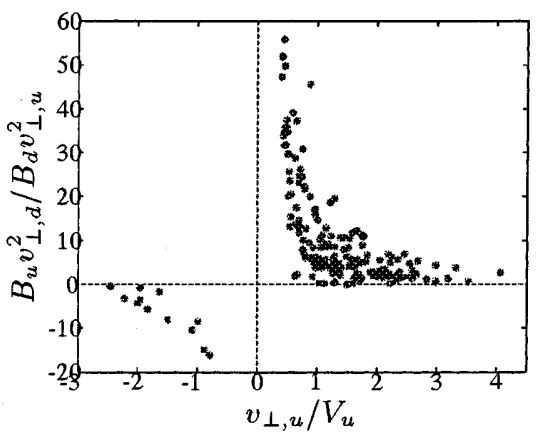

(b)

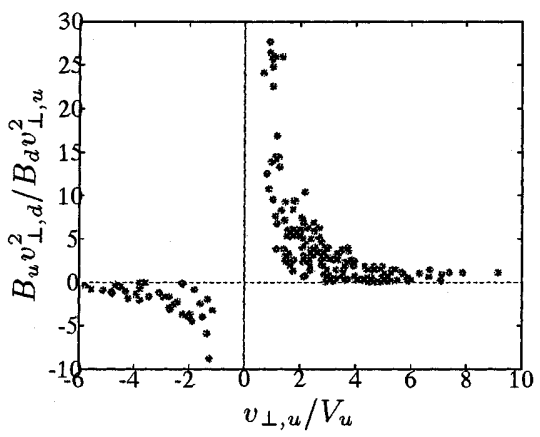

Fig. 6. Dependence of $B_{u} v_{\perp, d}^{2} / B_{d} v_{\perp, u}^{2}$ on $v_{\perp, u}$ for different angles and $\beta_{e}: \mathbf{a} \theta=80^{\circ}, \beta_{e}=0.05$; b $\theta=60^{\circ}, \beta_{e}=0.05 ;$ c $\theta=80^{\circ}, \beta_{e}=0.25$; and d $\theta=60^{\circ}, \beta_{e}=0.25$. Negative values of $v_{\perp, u}$ are used to show separately the electrons which leak from downstream and those reflected (see explanation in the text) are reflected off the shock front because of their inability to overcome the magnetic barrier despite the accelerating electric field. They have negative final parallel velocities $v_{\|, f}<0$ after tracing. The electrons which initially have negative parallel velocities should have come from behind the shock, that is, leak from the downstream region. In order to separate these two groups of electrons from the transmitted ones we use in Fig. 6 for them negative values of $v_{\perp, u}$ (multiplying it by sign $\left.\left(v_{\|, f}\right)\right)$. Such electrons are absent for very low $\beta_{e}$ (plots a and c) but appear for $\beta_{e}=0.25$ (plots b and $\mathrm{d}$ ). It is clearly seen that the demagnetization rapidly enhances with the decrease of the angle $\theta$ and $\beta_{e}$ : (a) higher values of $K$ are achieved; and (b) more particles have $K>10$ (an order of magnitude higher than the adiabatic value $K=1$ ) for lower $\beta_{e}$ (with the same $\theta$ ) and smaller angle $\theta$ (with the same $\beta_{e}$ ). The weakest demagnetization is found for the case $\beta_{e}=0.25$ and $\theta=80^{\circ}: K \approx 1$ already for $v_{\perp, u} / V_{u} \geq 2.5 \approx v_{T e}$.

For comparison, Fig. 7 shows the same dependence for the adiabatic case, where for the transmitted electrons (those with $v_{\perp, u}>0$ ) one has $K=1$.

It is also of interest to compare the parts of the upstream electron distribution, for which demagnetization is strong, for different $\theta$ and $\beta$. Such comparison is presented in Fig. 8 where the electrons corresponding to the whole upstream distribution are shown by circles, while those electrons which have $B_{u} v_{\perp, d}^{2} / B_{d} v_{\perp, u}^{2}>2$ are shown by crosses. It is seen that the demagnetization always covers the central part of the distribution. For the same $\theta$ and different $\beta_{e}$ the demagnetized electrons are the same but the relative weight of the nonadiabatic part is smaller for higher $\beta_{e}$. For the same $\beta_{e}$ a larger part of the upstream electron distribution becomes demagnetized for more oblique geometry (smaller $\theta$ ).

To illustrate the dependence of the demagnetization on $\beta_{e}$ we present in Fig. 9 the ratio

$K_{T}=\frac{B_{u}\left\langle v_{\perp, d}^{2}\right\rangle}{B_{d}\left\langle v_{\perp, u}^{2}\right\rangle}=\frac{B_{u} \int_{>} v_{\perp, d}^{2} f_{d}\left(v_{\|, d}, v_{\perp, d}\right) \mathrm{d}^{3} \mathbf{v}_{d}}{B_{d} \int v_{\perp, u}^{2} f_{u}\left(v_{\|, u}, v_{\perp, u}\right) \mathrm{d}^{3} \mathbf{v}_{u}}$.

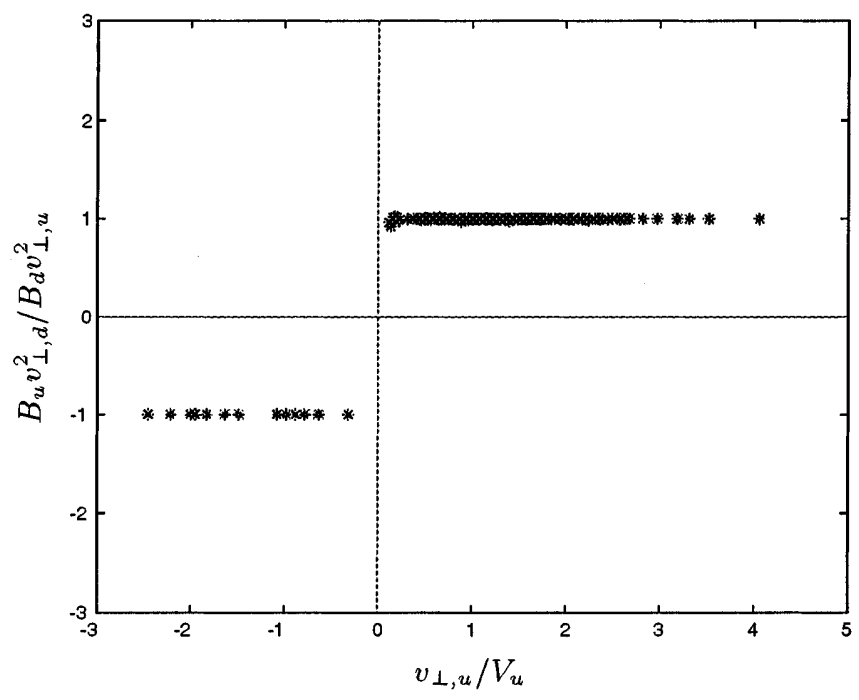

Fig. 7. Dependence of $B_{u} v_{\perp, d}^{2} / B_{d} v_{\perp, u}^{2}$ on $v_{\perp, u}$ in the adiabatic regime 

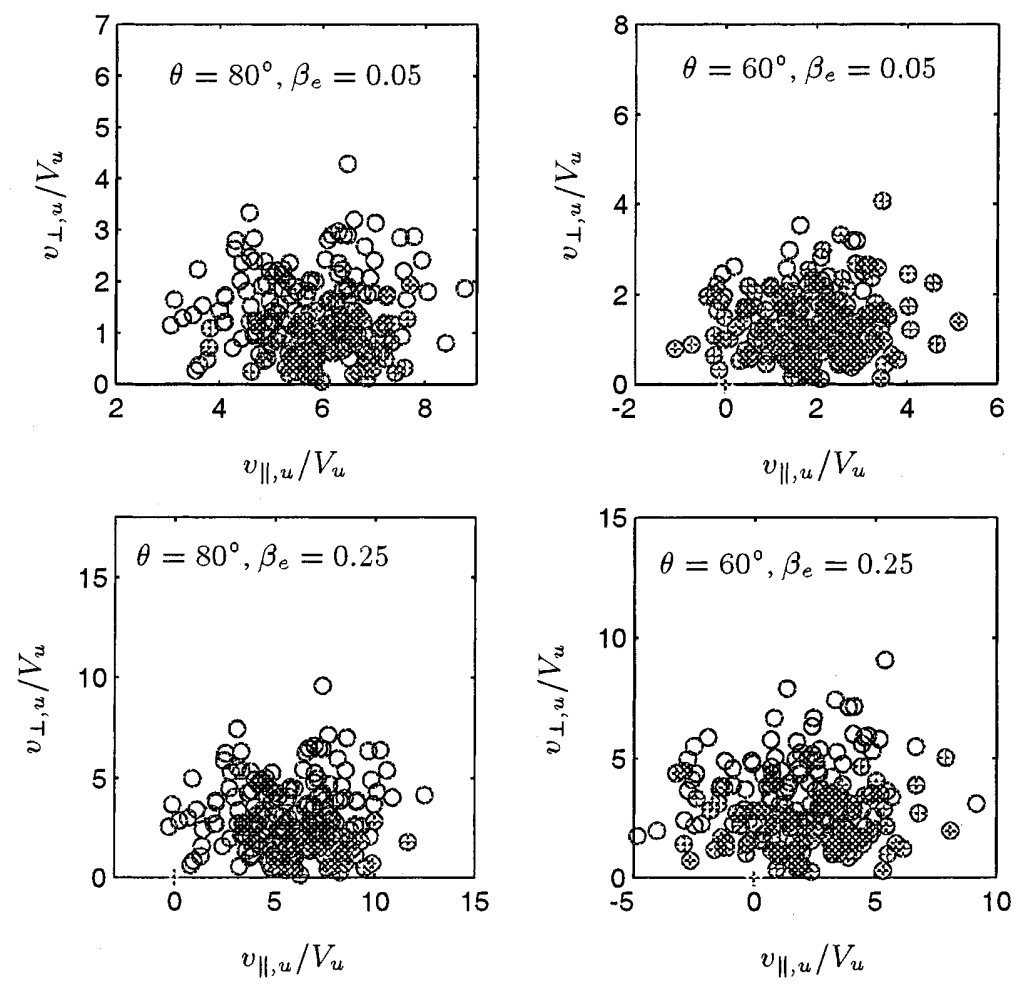

Fig. 8. Upstream electron distribution for different $\theta$ and $\beta_{e}$ (circles) and the part of it corresponding to $B_{u} v_{\perp, d}^{2} / B_{d} v_{\perp, U}^{2}>2$ (crosses)

The integral in the nominator is only over transmitted electrons with $v_{\|, d}>0$, and the ratio of two integrals has the meaning of the ratio of the downstream temperature of transmitted electrons to the upstream electron temperature. Numerical calculation of $K_{T}$ has been done using the relation $f_{d}\left(v_{\|, d}, v_{\perp, d}\right) \mathrm{d}^{3} \mathbf{v}_{d}=J\left(\mathbf{v}_{u}, \mathbf{v}_{d}\right) f_{u}$ $\left(v_{\|, u}, v_{\perp, u}\right) \mathrm{d}^{3} \mathbf{v}_{u}$, where the Jacobian of the transformation from the upstream to downstream velocities is (Gedalin, 1997) $J\left(\mathbf{v}_{u}, \mathbf{v}_{d}\right)=\left|v_{x, u} / v_{x, d}\right|$ in the stationary one-dimensional case.

The dependence is shown for $\theta=70^{\circ}$ (circles) and $\theta=60^{\circ}$ (stars), where the demagnetization is substantial for low $\beta_{e}$. It is seen that when $\beta_{e} \geq 0.5$, the distribution

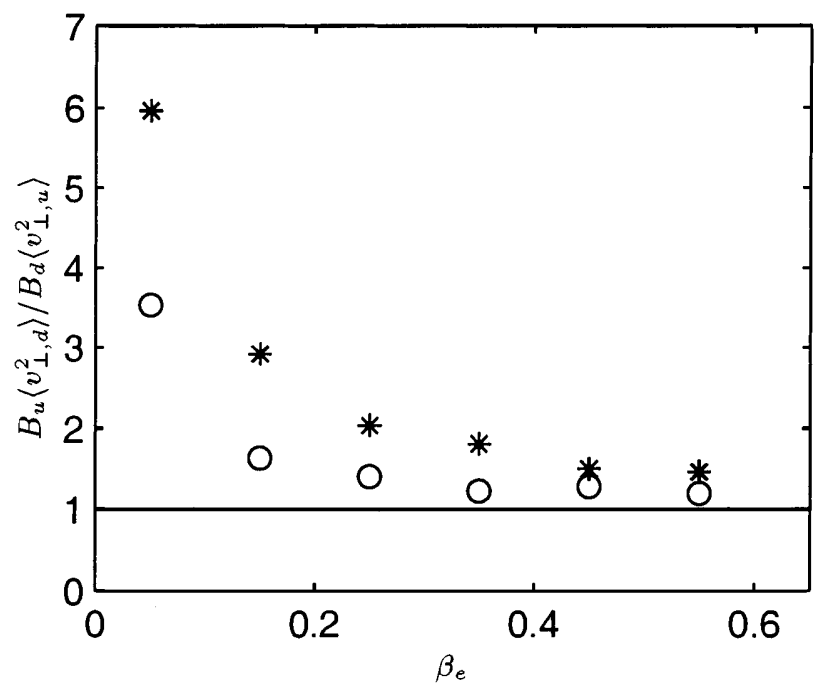

Fig. 9. Dependence of $B_{u}\left\langle v_{\perp, d}^{2}\right\rangle / B_{d}\left\langle v_{\perp, u}^{2}\right\rangle$ on $\beta_{e}$ for $\theta=70^{\circ}$ (circles) and $\theta=60^{\circ}$ (stars) behaves adiabatically. It should be emphasized that the electrons with low $v_{\perp, u}$ are still demagnetized, only their contribution in the downstream temperature becomes less important because of the decrease of their relative number when $\beta_{e}$ increases.

The above analysis may leave the impression that downstream electron distributions are gyrotropic. In order to show that this is not the case we perform the backward Liouville mapping. This is done by finding for each $\mathbf{v}_{d}$ (at $x=$ const) its progenitor $\mathbf{v}_{u}$ (using backward time integration) and applying the collisionless relation $f\left(\mathbf{v}_{d}\right)=f_{0}\left(\mathbf{v}_{u}\right)$, where $f_{0}\left(\mathbf{v}_{u}\right)$ is the known (Maxwellian) upstream distribution function. We define the angle $\phi$ between the downstream electron velocity and downstream magnetic field (the last one is in the $x z$ plane), so that $v_{\|, d}=\left|\mathbf{v}_{d}\right| \cos \phi, v_{\perp, d}=\left|\mathbf{v}_{d}\right| \sin \phi$. We also define the angle $\psi$ so that $\mathbf{v}_{\perp, \mathrm{d} x}=v_{\perp, d} \cos \psi$ and $\mathbf{v}_{\perp, \mathrm{d} y}=v_{\perp, d} \sin \psi$. Fig. 10 shows downstream distributions for several values of $\psi$ and $\phi=45^{\circ}$ for $\beta_{e}=0.05$ (strong demagnetization) and $\beta_{e}=0.25$ (weak demagnetization). Fluctuations of the individual distributions and difference between distributions for different $\psi$ (for the same $v_{d}$ and $\phi$ ) are much larger in the strong demagnetization case. The same is seen from Fig. 11 where the corresponding downstream distributions are shown for $\theta=80^{\circ}$ and $\beta_{e}=0.05$.

\section{Discussion and conclusion}

In the present paper we have studied the collisionless electron dynamics in a high Mach number shock with small-scale structure, when electrons become substantially demagnetized. We have analyzed the dependence 


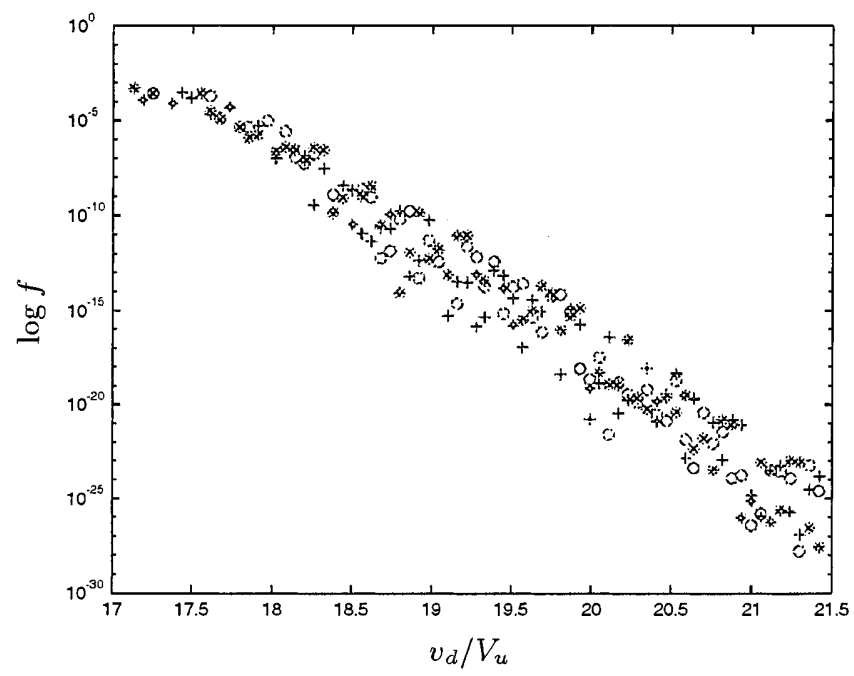

(a)

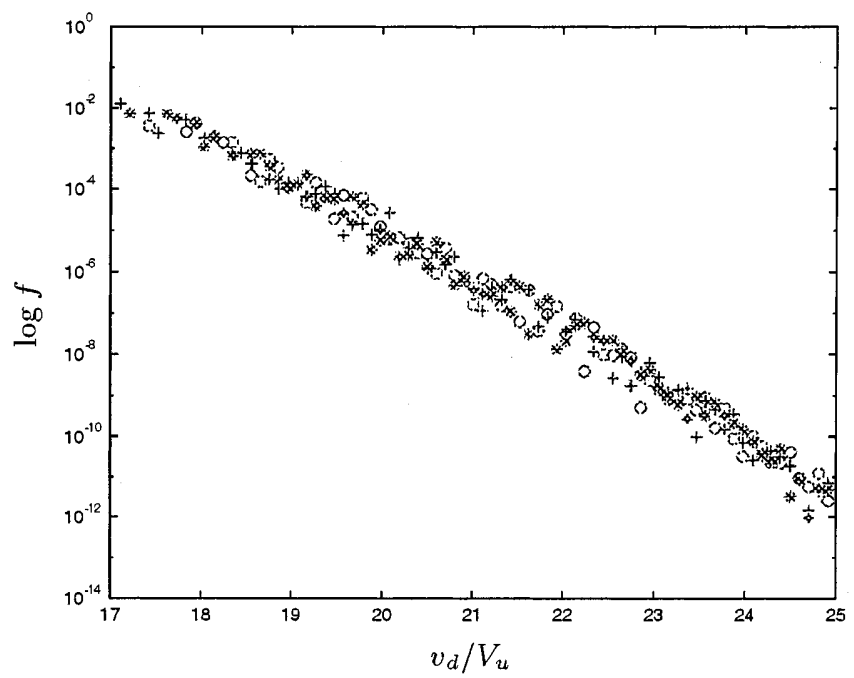

(b)

Fig. 10. Downstream electron distribution for $\phi=45^{\circ}$ and different values of $\psi=0^{\circ}, 90^{\circ}$, and $180^{\circ}$ (see explanation in the text). The shock angle $\theta=60^{\circ}$, while for $\mathbf{a} \beta_{e}=0.05$ and for $\mathbf{b} \beta_{e}=0.25$

of the demagnetization on the angle between the shock normal and upstream magnetic field and on the upstream $\beta_{e}$. The analysis have been carried out for a particular shock model, with the de Hoffman-Teller cross-shock potential typical for shocks with strong heating. There is the place to make several comments about the model and the analysis. First, the collisionless electron dynamics, whether adiabatic or nonadiabatic, is not able to describe properly the formation of the inner (low energy) part of the downstream electron distribution where a gap forms (Feldman, 1985; Veltri et al., 1990, 1992; Veltri and Zimbardo, 1993a, b; Hull et al., 1998; Gedalin and Griv, 1999). Therefore, it is impossible to make conclusions about the downstream electron temperature unless we know the mechanism

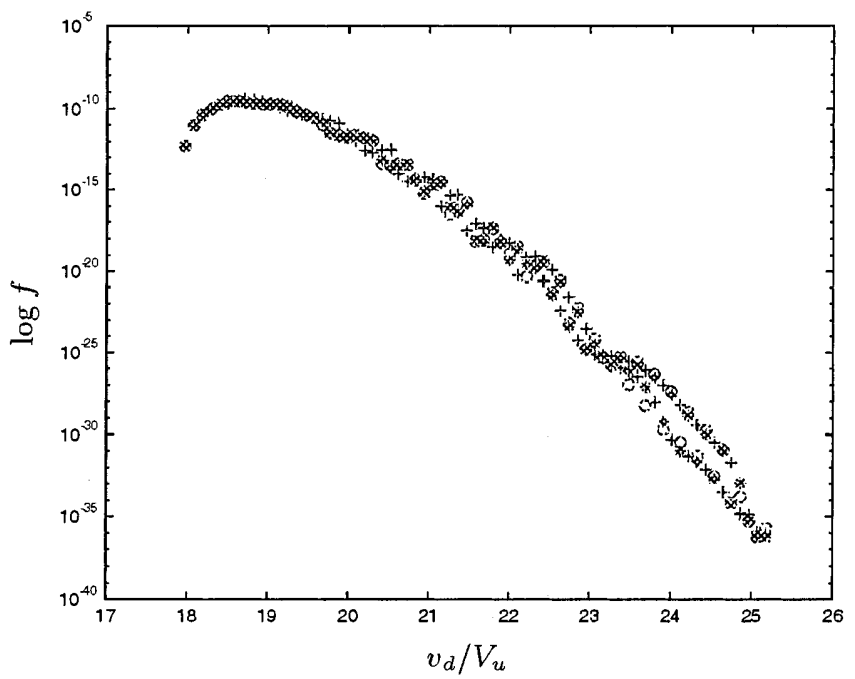

Fig. 11. Downstream electron distribution for $\phi=45^{\circ}$ and different values of $\psi=0^{\circ}, 90^{\circ}$, and $180^{\circ}$ (see explanation in the text). The shock angle $\theta=80^{\circ}$ and $\beta_{e}=0.05$

for the gap filling and the details of electron dynamics affect directly only the high energy tail. Second, the chosen shock model is hardly typical taking into account variety of shock profiles (Newbury et al., 1998). It is however typical in that that the small scale features have the width of $\sim 0.1\left(c / \omega_{p i}\right)$, where one could expect strong demagnetization. Third, we studied the dependence on the angle and $\beta_{e}$ assuming that all other shock parameters remain the same. Strictly speaking, the shock parameters should have been chosen consistently, but since details of the shock structure are not understood and correlations of the shock parameters are unknown yet there is no other way to study theoretically the electron dynamics in different conditions. It should be understood that the present analysis is not able to provide tools for direct comparison with observations nor can it provide estimates of electron heating unless we know the details of the shock smallscale structure. Nevertheless, it provides valuable information about the behavior of electrons in the quasistationary fields of in the shock front for different shock conditions.

To summarize, we have found that for fixed Mach number, magnetic compression, and cross-shock potential, electron demagnetization is stronger for stronger obliquity (smaller angle between the shock normal and upstream magnetic field). This may be the reason why the shocks with strongest electron heating are oblique. Demagnetization becomes weaker with the angle increase. On the other hand, when the angle is too small shocks become more quasiparallel and their typical scales are larger than those for quasiperpendicular shocks. Thus, electron demagnetization may be expected to be stronger for shocks with $\theta \approx 60-70^{\circ}$ and not in nearly perpendicular shocks, in agreement with observations (Schwartz et al., 1988).

We have shown also that for larger $\beta_{e}$ (which corresponds to higher upstream electron temperatures for fixed Mach number) demagnetization is less pro- 
nounced. This finding is in agreement with observations (Schwartz et al., 1988) showing weakening of electron heating with the decrease of $V_{u} / v_{T e}$. We have shown also that the downstream electron distribution is substantially non-gyrotropic in the strong demagnetization case. Although this non-gyrotropy cannot be measured by a spacecraft performing averaging over some spatial region (as happens for ISEE and AMPTE measurements) it may be important for development of additional local microinstabilities which can smooth out and isotropize the electron distribution.

Acknowledgements. The research was supported in part by Israel Science Foundation under grant No. 261/96-1.

Topical Editor K.-H. Glassmeier thanks L. Ball and another referee for their help in evaluating this paper.

\section{References}

Balikhin, M., and M. Gedalin, Kinematic mechanism for shock electron heating: comparison of theoretical results with experimental data, Geophys. Res. Lett., 21, 841, 1994.

Balikhin, M., M. Gedalin, and A. Petrukovich, New mechanism for electron heating in shocks, Phys. Rev. Lett., 70, 1259, 1993.

Balikhin, M., V. V. Krasnosel'skikh, L. J. C. Woolliscroft, and M. Gedalin, A study of the dispersion of the electron distribution in the presence of $E$ and $B$ gradients: application to electron heating at quasi-perpendicular shocks, J. Geophys. Res., 103, 2029, 1998.

Ball, L., and D. Galloway, Electron heating by the cross-shock electric potential, J. Geophys. Res., 103, 17,455, 1998.

Feldman, W. C., Electron velocity distributions near collisionless shocks, in Collisionless Shocks in the Heliosphere: Reviews of Current Research, Geo phys. Monogr. Ser., vol. 35, ed. by R.G. Stone and B.T. Tsurutani, p. 195, AGU, Washington, D.C., 1985.

Feldman, W. C., S. J. Bame, S. P. Gary, J. T. Gosling, D. McComas, M. F. Thomsen, G. Paschmann, N. Sckopke, M. M. Hoppe, and C. T. Russell, Electron heating within the earth's bow shock, Phys. Rev. Lett., 49, 199, 1982.

Gedalin M., Noncoplanar magnetic field in the collisionless shock front, J. Geophys. Res., 101, 11,153, 1996.

Gedalin, M., Ion heating in oblique low-Mach number shocks, Geophys. Res. Lett., 24, 2511, 1997.

Gedalin, M., Two-stream instability of electrons in the shock front, Geophys. Res. Lett., 26, 1239, 1999.

Gedalin, M., and M. Balikhin, Width dependent collisionless electron dynamics in the static field of the shock ramp, 1, Single particle behavior and implications for downstream distribution, Nonl. Proc. Geophys., 4, 167, 1998.

Gedalin, M., and E. Griv, Role of overshoots in the formation of the downstream distribution of adiabatic electrons, J. Geophys. Res., 104, 14821, 1999.

Gedalin, M., K. Gedalin, M. Balikhin, V. Krasnosselskikh, and L. J. C. Woolliscroft, Demagnetization of electrons in inhomogeneous $\mathbf{E} \perp \mathbf{B}$ : implications for electron heating in shocks, J. Geophys. Res., 100, 19,911, 1995a.
Gedalin, M., K. Gedalin, M. Balikhin, and V. V. Krasnoselskikh, Demagnetization of electrons in the electromagnetic field structure, typical for oblique collisionless shock front, J. Geophys. Res., 100, 9481, 1995 b.

Gedalin, M., U. Griv, and M. Balikhin, Width dependent collisionless electron dynamics in the static field of the shock ramp, 2, Phase space portrait, Nonl. Proc. Geophys., 4, 173, 1998a.

Gedalin, M., J. A. Newbury, and C. T. Russell, Numerical analysis of collisionless particle motion in an observed supercritical shock front, Eos. Trans. AGU 79(45), Fall Meet. Suppl., F173, 1998b.

Goodrich, C. C., and J. D. Scudder, The adiabatic energy change of plasma electrons and the frame dependence of the cross shock potential at collisionless magnetosonic shock waves, J. Geophys. Res., 89, 6654, 1984.

Hull, A. J., J. D. Scudder, L. A. Frank, W. R. Paterson, and M. G. Kivelson, Electron heating and phase space signatures at strong and weak quasiperpendicular shocks, J. Geophys. Res., 103, 2041, 1998.

Jones, F. C., and D. C. Ellison, Noncoplanar magnetic fields, shock potentials, and ion deflection, J. Geophys. Res., 92, 11205, 1987

Jones, F. C. and D. C. Ellison, The plasma physics of shock acceleration, Space Sci. Rev., 58, 259, 1991.

Newbury, J. A., and C. T. Russell, Observations of a very thin collisionless shock, Geophys. Res. Lett., 23, 781, 1996.

Newbury, J. A., C. T. Russell, and M. Gedalin, The determination of shock ramp width using the noncoplanar magnetic field component, Geophys. Res. Lett., 24, 1975, 1997.

Newbury, J. A., C. T. Russell, and M. Gedalin, The ramp widths of high Mach number, quasi-perpendicular collisionless shocks, J. Geophys. Res., 103, 29,581, 1998.

Schwartz, S. J., M. F. Thomsen, S. J. Bame, and J. Stansbury, Electron heating and the potential jump across fast mode shocks, J. Geophys. Res., 93, 12,923, 1988.

Scudder, J. D., A review of the physics of electron heating at collisionless shocks, Adv. Space Res., 15, 181, 1995.

Scudder, J. D., A. Mangeney, C. Lacombe, C. C. Harvey, and T. L. Aggson, The resolved layer of a collisionless, high $\beta$, supercritical, quasiperpendicular shock wave, 2, Dissipative fluid electrodynamics, and stationarity, J. Geophys. Res., 91, 11,053, 1986a.

Scudder, J. D., A. Mangeney, C. Lacombe, C. C. Harvey, C. S. Wu, and R. R Anderson, The resolved layer of a collisionless, high $\beta$, supercritical quasiperpendicular shock wave, 3, Vlasov electrodynamics, J. Geophys. Res., 91, 11,075, 1986b.

Thomsen, M. F., M. M. Mellott, J. A. Stansbury, S. J. Bame, J. T. Gosling, and C. T. Russell, Strong electron heating at the Earth's bow shock, J. Geophys. Res., 92, 10,119, 1987.

Veltri, P., and G. Zimbardo, Electron-whistler interaction at the Earth's bow shock. 1. Whistler instability, J. Geophys. Res., 98, 13,325, 1993a.

Veltri, P., and G. Zimbardo, Electron-whistler interaction at the Earth's bow shock: 2, Electron pitch angle diffusion, J. Geophys. Res., 98, 13,335, 1993b.

Veltri, P., A. Mangeney, and J. D. Scudder, Electron heating in quasiperpendicular shocks: a Monte-Carlo simulation, J. Geophys. Res., 95, 14,939, 1990.

Veltri, P., A. Mangeney, and J. D. Scudder, Reversible electron heating vs. wave-particle interactions in quasi-perpendicular shocks, Nuovo Cimento, 15C, 607, 1992. 